\title{
Eficiência Produtiva em Vacas Primíparas das Raças Aberdeen Angus e Charolês
}

\author{
Edson Luis de Azambuja Ribeiro', João Restle², Marco Antônio da Rocha1, \\ Ivone Yurika Mizubuti ${ }^{1}$, Leandro das Dôres Ferreira da Silva ${ }^{1}$
}

\begin{abstract}
RESUMO - Este trabalho foi conduzido com o objetivo de avaliar o desempenho produtivo de vacas de corte. Foram utilizadas 30 vacas da raça Aberdeen Angus e 32 da raça Charolês primíparas, prenhes no início do experimento, com bezerros puros ou mestiços Nelore. As vacas foram submetidas a diferentes tratamentos alimentares durante o inverno e a primavera: T1 - pastagem natural, T2 - pastagem cultivada por 60 dias (24 horas/dia), do início de setembro ao início de novembro, T3 - acesso à pastagem cultivada por duas horas diárias, por um período de 60 dias (de início de julho ao início de setembro), T4 - acesso à pastagem cultivada por duas horas diárias, por um período de 60 dias (de início de julho ao início de setembro), e mais 60 dias (24 horas/dia) de pastagem cultivada do início de setembro ao início de novembro. As medidas de eficiência produtiva foram: EPPARTO = $(\mathrm{P} 205 / \mathrm{PVP}) * 100 ;$ EPDESMAME $=(\mathrm{P} 205 / \mathrm{PVD}) * 100$; EPMBPARTO $=\left(\mathrm{P} 205 / \mathrm{PVP}^{0,75}\right) ;$ EPMBDESMAME $=\left(\mathrm{P} 205 / \mathrm{PVD}^{0,75}\right) ;$ e EPNDT $=$ NDTTOTAL/P205, em que P205 é o peso ao desmame dos bezerros; PVP e PVD, os pesos das vacas ao parto e ao desmame, respectivamente; e NDTTOTAL, a exigência em energia para manutenção e produção de leite das vacas. As vacas Aberdeen Angus, com bezerros machos e aquelas com bezerros mestiços, foram mais eficientes. Vacas que utilizaram a pastagem cultivada por um período de tempo maior (T4) tiveram melhor desempenho do que aquelas que permaneceram apenas em pastagem natural(T1); as vacas dos outros tratamentos tiveram desempenhos intermediários.
\end{abstract}

Palavras-chave: cruzamento, gado de corte, leite, peso, produtividade

\section{Productive Efficiency of Angus and Charolais Primiparous Cows}

\begin{abstract}
The objective of this work was to evaluate the productive efficiency of beef cows. Thirty Angus and 32 Charolais primiparous cows, pregnant at the beginning of the experiment with straightbred or crossbred calves, were evaluated. The cows were submitted to different feeding management during winter and spring: T1 - Native pasture, T2 - Cultivated pasture for 60 days (24 hours/ day), from early September to early November, T3 - Cultivated pasture for two hours a day, for 60 days, from early July to early September, T4 - Cultivated pasture for two hours a day, for 60 days, from early July to early September, and more 60 days on cultivated pasture (24 hours/day), from early September to early November. Productive efficiency was measured by CALVINGPE $=($ W205/ $\mathrm{CWC}) * 100$, WEANINGPE $=(\mathrm{W} 205 / \mathrm{CWW}) * 100$, CALVINGMBPE $=\left(\mathrm{W} 205 / \mathrm{CWC}^{\cdot 75}\right)$, WEANINGMBPE $=(\mathrm{W} 205 / \mathrm{CWW} \cdot 75)$ and TDNPE $=$ TDNTOTAL/W205, where W205 is the calf weaning weight and CWC and CWW are the cow weight at calving and weaning, respectively. TDNTOTAL is the total energy requirement (maintenance + milk production) of the cow. Aberdeen Angus cows, cows with male calves and cows with crossbred calves were more efficient. Cows that had access to cultivated pasture for a long period of time (T4) had better performance than those that were maintained on native pasture only (T1). Cows of the other treatments had intermediate performance.
\end{abstract}

Key Words: beef cattle, crossbreeding, milk, productivity, weight

\section{Introdução}

A eficiência produtiva em bovinos de corte, na fase de cria, está diretamente relacionada com o desempenho reprodutivo das fêmeas, com a sua habilidade materna e com o potencial de ganho de peso dos bezerros. Outro fator que nem sempre é levado em consideração, mas é de suma importância na viabilidade da atividade, é o requerimento total de nutrientes.

Geralmente, as vacas maiores dentro de uma raça ou entre raças ou cruzamentos produzem bezerros mais pesados ao desmame, porém têm maiores requerimentos de manutenção e, normalmente, produzem mais leite, o que aumenta os seus requerimentos nutricionais (EUCLIDES FILHO et al., 1984; KRESS et al., 1990; PRICHARD e MARSHALL, 1993). Segundo MONTAÑO-BERMUDEZ et al. (1990), vacas de mesmo tamanho e que produzem mais leite, além do maior requerimento para a produção de leite, têm maiores requerimentos para manutenção.

Várias medidas de eficiência de um rebanho ou de animais individualmente têm sido descritas na

\footnotetext{
1 Professor Associado do Departamento de Zootecnia da Universidade Estadual de Londrina. 86051-970, Londrina, PR. E.mail:elar@npd.uel.br

2 Professor Titular, Departamento de Zootecnia da Universidade Federal de Santa Maria.
} 
literatura. Estas medidas, apesar de serem avaliadas por diferentes metodologias de cálculo, procuram levar em consideração as diferenças em peso (ou tamanho) e ou requerimentos nutricionais dos animais. Para essas medidas são utilizadas as relações entre os pesos dos bezerros ao desmame, ou ao abate, com os pesos vivo ou metabólico das vacas ao parto, início do acasalamento ou ao desmame dos bezerros, bem como com o consumo total de energia (KRESS et al., 1990; MONTAÑO-BERMUDEZ e NIELSEN, 1990; EUCLIDES FILHO et al., 1995; ALENCAR et al., 1999ab). Além destas características, dados de desempenho reprodutivo devem complementar as medidas de eficiência produtiva (EUCLIDES FILHO et al., 1984; KRESS et al., 1990; BARCELLOS et al., 1996). Considerando o ambiente em que os animais são mantidos e o grupo genético, vacas maiores, que produzem bezerros mais pesados ao desmame, podem ser mais eficientes (BARCELLOS et al., 1996) ou não (EUCLIDES FILHO et al., 1984; KRESS et al., 1990).

A alimentação no pré e pós-parto é importante para o desempenho produtivo do gado de cria, sendo que o peso da vaca ao parto e ou início do acasalamento, relacionados com a condição corporal da vaca, tem reflexos sobre o desempenho reprodutivo, principalmente com o intervalo parto-cio e com a taxa de gestação (SIMEONE e LOBATO, 1996; BUSTAMANTE et al., 1997; LOBATO et al., 1998). Melhor condição de alimentação nestas fases melhora também o desempenho dos bezerros, em função de maior produção de leite das vacas (QUADROS e LOBATO, 1997).

O objetivo deste trabalho foi comparar diferentes medidas de eficiência produtiva em vacas primíparas das raças Charolês e Aberdeen Angus, com bezerros puros ou mestiços ao pé, e submetidas a diferentes planos de suplementação no inverno e primavera.

\section{Material e Métodos}

O experimento foi conduzido na fazenda experimental do Departamento de Zootecnia da Universidade Federal de Santa Maria, Santa Maria, RS. Foram utilizadas 62 vacas primíparas com cria ao pé, com idade média de quatro anos, sendo 30 vacas da raça Aberdeen Angus e 32 da raça Charolês. Parte dos bezerros das vacas da raça Aberdeen Angus era representada por animais puros $(n=12)$ e a outra, por mestiços Nelore (1/2Nelore-1/2Aberdeen Angus; $\mathrm{n}=18$ ), o mesmo ocorrendo para os das vacas da raça
Charolês, em que metade foi constituída por animais puros $(n=16)$ e a outra metade $(n=16)$, por mestiços Nelore (1/2Nelore-1/2Charolês).

No final do período de gestação e início da lactação, as vacas foram submetidas a quatro tratamentos alimentares, sendo:

T1 - Pastagem natural;

T2 - Pastagem cultivada por 60 dias (24 horas/dia), do início de setembro ao início de novembro;

T3 - Vacas em pastagem natural, com acesso à pastagem cultivada por duas horas diárias, por um período de 60 dias (de início de julho ao início de setembro);

T4 - Vacas em pastagem natural, com acesso à pastagem cultivada por duas horas diárias, por um período de 60 dias (de início de julho ao início de setembro), e mais 60 dias (24 horas/dia) de pastagem cultivada do início de setembro ao início de novembro.

Os tratamentos foram delineados para atender os períodos tidos como de maior exigência nutricional por parte das vacas, final de gestação (T3), início de lactação (T2), e ambos os períodos (T4), sendo o T1 o testemunha. A área comum de pastagem natural, onde os animais permaneceram a maior parte do tempo, era constituída por gramíneas características da região fisiográfica da Depressão Central do Rio Grande do Sul. A região apresenta clima subtropical úmido (Cfa), segundo a classificação de Köppen. A lotação média utilizada nesta área foi de 0,9 vacas por hectare. A pastagem cultivada foi composta de uma mistura de azevém (Lolium multiflorum) e aveia (Avena strigosa). Nesta pastagem utilizou-se lotação flutuante de acordo com a disponibilidade da mesma. Maiores detalhes sobre as disponibilidades e características qualitativas das pastagens foram apresentadas por RIBEIRO et al. (1990).

As características avaliadas neste experimento foram peso da vaca nas primeiras 24 após o parto (PVP) e ao desmame dos bezerros (PVD), peso dos bezerros ajustados para 205 dias de idade (P205), produção média diária de leite das vacas, obtida por ordenha manual (RIBEIRO et al., 1991), e porcentagem média de gordura do leite. As produções médias diárias de leite foram corrigidas para $4 \%$ de gordura pela fórmula LEITE4\% $=(0,4+0,15 \mathrm{X}) \mathrm{L} 1$, em que L1 é a produção a ser corrigida e "X", o teor de gordura original.

As estimativas das medidas de eficiência produtiva foram obtidas a partir das características quantificadas . A primeira estimativa de eficiência foi 
considerada como a quantidade de quilos de bezerros desmamados para cada 100 quilos de vacas paridas: EPPARTO $=(\mathrm{P} 205 / \mathrm{PVP}) * 100$. Na segunda estimativa, substituiu-se o peso da vaca ao parto pelo peso da vaca ao desmame, sendo: EPDESMAME $=(\mathrm{P} 205 /$ PVD)*100. Para estimar a terceira medida de eficiência produtiva, baseou-se no requerimento de energia da vaca, expresso em quilos de NDT, para produzir um quilograma de bezerro desmamado: EPNDT $=$ NDTTOTAL/P205, em que NDTTOTAL é a somatória do requerimento para manutenção, tendo como base o peso da vaca ao parto e do requerimento para produção de leite até o desmame. Para determinar o requerimento para produção de leite, baseou-se na produção e no teor de gordura do leite, utilizando-se as tabelas do NATIONAL RESEARCH COUNCIL - NRC, segundo CAMPOS (1995). A quarta medida de eficiência foi estimada por meio da quantidade de quilos de bezerros desmamados por unidade de peso metabólico ao parto das vacas: $\mathrm{EPMBPARTO}=\mathrm{P} 205 /(\mathrm{PVP})^{0,75} \mathrm{e}$, na última medida de eficiência, o peso da vaca ao parto foi substituído pelo peso da vaca ao desmame, sendo: EPMBDESMAME $=$ P205/(PVD $)^{0,75}$.

Os resultados foram submetidos à análise de variância (SAS, 1989), tendo como efeitos indepen- dentes o grupo genético do bezerro, o sexo, o tratamento e as interações duplas entre estes efeitos. As diferenças entre médias foram detectadas a $5 \%$ de probabilidade (PDIFF). Estudo de contrastes foram realizados para comparar os grupos genéticos Aberdeen Angus (puros + mestiços) e Charolês (puros + mestiços) e puros (Aberdeen Angus + Charolês) com mestiços ((1/2Nelore- $1 / 2 \mathrm{~A}$. Angus + $1 / 2$ Nelore-1/2Charolês).

\section{Resultados e Discussão}

Não houve interação $(P>0,05)$ entre os efeitos para todas as características avaliadas. Na Tabela 1 , são apresentados os pesos das vacas ao parto e ao desmame, a produção média diária de leite ajustada para $4 \%$ de gordura, o peso dos bezerros ajustados para 205 dias de idade e o requerimento em NDT das vacas do parto ao desmame, de acordo com o grupo genético do bezerro e do sexo. Todas estas características foram afetadas pelo grupo genético do bezerro $(\mathrm{P}<0,05)$, porém, apenas o peso do bezerro ao desmame foi influenciado pelo sexo, em que os machos foram $6,9 \%$ mais pesados que as fêmeas.

As vacas da raça Charolês, amamentando bezerros puros como mestiços Nelore, foram significativa-

Tabela 1 - Médias estimadas e erros-padrão para pesos das vacas ao parto (PVP) e ao desmame (PVD), peso dos bezerros ao desmame (P205), produção média diária de leite ajustada para $4 \%$ de gordura e requerimento de energia das vacas do parto até o desmame (NDTTOTAL), segundo o sexo e o grupo genético do bezerro

Table 1 - Least square means and standard errors for cow weight at calving (CWC) and at weaning (CWW), calves weaning weight (W205), daily average milk production adjusted to $4 \%$ of fat and cow energy requirement from calving to weaning (TDNTOTAL), according to sex and genetic group of calves

\begin{tabular}{|c|c|c|c|c|c|}
\hline Item & $\begin{array}{l}\mathrm{PVP}, \mathrm{kg} \\
\quad C W C\end{array}$ & $\begin{array}{l}\mathrm{PVD}, \mathrm{kg} \\
C W W\end{array}$ & $\begin{array}{l}\mathrm{P} 205, \mathrm{~kg} \\
W 205\end{array}$ & $\begin{array}{l}\text { LEITE- } 4 \% \text {, litros } \\
\text { MILK-4\%, liters }\end{array}$ & $\begin{array}{c}\text { NDTTOTAL, } \mathrm{kg} \\
\text { TDNTOTAL }\end{array}$ \\
\hline \multicolumn{6}{|c|}{$\begin{array}{l}\text { Grupo genético do bezerro } \\
\text { Calf genetic group }\end{array}$} \\
\hline $\begin{array}{l}\text { Aberdeen Angus (A) } \\
\text { Angus (A) }\end{array}$ & $290,5 \pm 14,0^{\mathrm{a}}$ & $309,4 \pm 13,0^{\mathrm{a}}$ & $123,7 \pm 5,6^{\mathrm{a}}$ & $2,9 \pm 0,3^{\mathrm{a}}$ & $707,9 \pm 30,8^{a}$ \\
\hline $\begin{array}{l}\text { Charolês }(\mathrm{C}) \\
\text { Charolais }(C)\end{array}$ & $415,9 \pm 10,9^{b}$ & $411,9 \pm 10,1^{b}$ & $138,6 \pm 4,3^{b c}$ & $3,3 \pm 0,2^{\mathrm{ab}}$ & $913,3 \pm 23,9^{b}$ \\
\hline $\begin{array}{l}\text { Nelore } \times \mathrm{A} \\
\text { Nellore } \times A\end{array}$ & $322,8 \pm 9,9^{a}$ & $313,5 \pm 9,2^{\mathrm{a}}$ & $131,8 \pm 4,0^{\mathrm{ab}}$ & $3,1 \pm 0,2^{\mathrm{a}}$ & $770,2 \pm 21,7^{\mathrm{a}}$ \\
\hline $\begin{array}{l}\text { Nelore } \times \mathrm{C} \\
\text { Nellore } \times C\end{array}$ & $419,5 \pm 10,2^{b}$ & $398,6 \pm 9,5^{b}$ & $147,5 \pm 4,1^{\mathrm{c}}$ & $3,8 \pm 0,2^{b}$ & $951,0 \pm 22,5^{\mathrm{b}}$ \\
\hline $\begin{array}{l}\text { Sexo do bezerro } \\
\text { Calf sex }\end{array}$ & & & & & \\
\hline $\begin{array}{l}\text { Macho } \\
\text { Male }\end{array}$ & $361,3 \pm 8,1$ & $353,9 \pm 7,5$ & $139,9 \pm 3,2^{\mathrm{a}}$ & $3,3 \pm 0,2$ & $832,6 \pm 17,8$ \\
\hline $\begin{array}{l}\text { Fêmea } \\
\text { Female }\end{array}$ & $363,0 \pm 7,7$ & $362,8 \pm 7,1$ & $130,9 \pm 3,1^{\mathrm{b}}$ & $3,3 \pm 0,2$ & $838,6 \pm 16,8$ \\
\hline
\end{tabular}

Médias, na coluna, para o mesmo item, seguidas por letras diferentes são diferentes $(P<0,05)$ pelo teste t.

Means, within a column, for the same item, followed by different letters are different $(P<.05)$ by $t$ test. 
mente mais pesadas ao parto e ao desmame do que as vacas da raça Aberdeen Angus, amamentando bezerros puros ou mestiços Nelore. Em relação à produção de leite, as vacas da raça Charolês que amamentaram bezerros mestiços produziram mais leite $(\mathrm{P}<0,05)$ que as da raça Aberdeen Angus, porém não diferiram das vacas Charolês com bezerros puros, e estas, por sua vez, não diferiram das vacas Aberdeen Angus.

O estudo de contrastes mostrou que vacas da raça Charolês, com bezerros puros e mestiços, produziram mais leite que as vacas da raça Aberdeen Angus (3,6 vs 3,0 litros de leite por dia). Porém, apesar de uma média aparentemente maior para produção de leite, o estudo de contrastes não detectou diferença significativa entre as vacas (Charolês + Aberdeen Angus) com bezerros mestiços e com bezerros puros (3,5 vs 3,1 litros por dia). As exigências em NDT foram maiores para as vacas da raça Charolês, em função do seu maior peso e produção leiteira.

Dados de produção de leite para vacas de corte variam com as raças ou cruzamentos, ambientes a que estão submetidas, nutrição, entre outros. MELTON et al. (1967), também, observaram maior produção diária em vacas da raça Charolês $(4,5 \mathrm{~kg})$ do que em vacas da raça Aberdeen Angus (3,8 kg). LEAL e FREITAS (1982) observaram produções (3,6 kg), para vacas da raça Charolês, semelhantes as observadas neste trabalho. As produções para as vacas da raça Aberdeen Angus são próximas às observadas (3,3 kg) por REYNOLDS et al. (1978) e são menores que a produção encontrada $(5,3 \mathrm{~kg})$ por EUCLIDES FILHO et al. (1984). ALBUQUERQUE et al. (1993) citaram produções de 1,98;3,08 e; $5,26 \mathrm{~kg}$ por dia, para vacas das raças Gir, Nelore e Caracú, respectivamente. Os valores observados em outros trabalhos, com outras raças ou cruzamentos, geralmente, são maiores que a média de 3,3 kg observada neste trabalho (EUCLIDES FILHO et al., 1984; KRESS et al., 1990; MONTAÑO-BERMUDEZ et al., 1990; CRUZ et al., 1997; QUADROS e LOBATO, 1997). Produções similares entre vacas amamentando machos e fêmeas foram observadas por KRESS et al. (1990) e ALBUQUERQUE et al. (1993). REYNOLDS et al. (1978) já haviam relatado que vacas com bezerros mestiços produzem mais leite, provavelmente por estes amamentarem com maior frequiência, o que estimularia maior produção por suas mães.

Em relação ao peso dos bezerros, observou-se que os mestiços $1 / 2$ Nelore-1/2 Charolês foram os mais pesados e os Aberdeen Angus, os mais leves ( $\mathrm{P}<0,05$; Tabela 1). O estudo de contrastes mostrou que os bezerros do grupo Charolês (mestiços + puros) foram mais pesados que os do grupo Aberdeen Angus, com médias respectivamente de 143 e $128 \mathrm{~kg}$. Na comparação entre bezerros mestiços e puros, observou-se que os mestiços pesaram 9,0 kg a mais que os puros; esta diferença esteve próxima de ser estatisticamente significativa $(\mathrm{P}=0,07)$.

Os cruzamentos em bovinos de corte, bem como a complementaridade entre raças, têm sido utilizados para explorar as heteroses materna e individual. $\mathrm{O}$ maior peso dos mestiços, neste trabalho, evidenciou que os últimos dois aspectos foram importantes. $\mathrm{O}$ maior peso dos bezerros machos corrobora os obtidos por vários autores (KRESS et al., 1990; ALBUQUERQUE et al., 1993; EUCLIDES FILHO et al., 1995; ALENCAR et al., 1997; ALENCAR et al., 1999b) e está relacionado, provavelmente, com a ação dos hormônios sexuais masculinos, que possuem ação anabolizante protéica. Geralmente, os machos já são mais pesados ao nascer, e esta diferença é mantida, ou aumentada, até a idade adulta. Os bezerros nesse experimento foram $13 \%$ mais pesados ao nascimento que as bezerras (RIBEIRO e RESTLE, 1991); aos 205 dias está diferença foi de $7 \%$.

Na Tabela 2, são apresentados os resultados para as medidas de eficiência produtiva, segundo o grupo genético do bezerro e o sexo. Pode-se observar que as vacas da raça Aberdeen Angus com bezerros mestiços Nelore ou com bezerros puros apresentaram, de modo geral, as melhores médias. O estudo de contrastes mostrou diferença entre as médias do grupo Aberdeen Angus (puros + mestiços) e o grupo Charolês, para todas as medidas de eficiência produtiva.

As vacas da raça Aberdeen Angus desmamaram, em média, 21,5\% mais quilos de bezerro para cada $100 \mathrm{~kg}$ de vaca ao parto (42,0 vs $34,6 \mathrm{~kg}$ ) e $15,7 \%$ mais quilos de bezerro para cada $100 \mathrm{~kg}$ de vacas ao desmame (41,2 vs 35,6 kg). Contudo, apesar de as vacas da raça Aberdeen Angus produzirem mais quilos de bezerros, tiveram exigência em NDT 11,4\% menor que as vacas Charolês $(5,85$ vs $6,60 \mathrm{~kg}$ ). Quando se usou, como medida de eficiência, a relação do peso do bezerro ao desmame, pelo peso metabólico da vaca ao parto ou peso metabólico da vaca ao desmame, observou-se que as vacas da raça Aberdeen Angus produziram 12,9 e 9,4\% mais quilos de bezerros por unidade de peso metabólico.

Quando se compararam vacas que amamentaram bezerros mestiços (1/2Nelore-1/2Charolês $+1 / 2$ Nelore- 
Rev. bras. zootec.

Tabela 2 - Médias estimadas e erros-padrão para medidas de eficiência produtiva ${ }^{1}$ de vacas de corte, segundo o sexo e o grupo genético do bezerro

Table 2 - Least square means and standard errors for measurements of productive efficiency ${ }^{1}$ of beef cows, according to sex and genetic group of the calves

\begin{tabular}{|c|c|c|c|c|c|}
\hline Item & $\begin{array}{l}\text { EPPARTO, } \mathrm{kg} \\
C A L V I N G P E\end{array}$ & $\begin{array}{l}\text { EPDESMAME, } \mathrm{kg} \\
\text { WEANINGPE }\end{array}$ & $\begin{array}{c}\text { EPNDT, } \mathrm{kg} \\
\text { TDNPE } \\
\end{array}$ & $\begin{array}{l}\text { EPMBPARTO } \\
C A L V I N G M B P E\end{array}$ & $\begin{array}{l}\text { EPMBDESMAME } \\
\text { WEANINGMBPE }\end{array}$ \\
\hline \multicolumn{6}{|c|}{$\begin{array}{l}\text { Grupo genético do bezerro } \\
\text { Calf genetic group }\end{array}$} \\
\hline $\begin{array}{l}\text { Aberdeen Angus (A) } \\
\text { Angus (A) }\end{array}$ & $42,9 \pm 1,8^{\mathrm{a}}$ & $40,1 \pm 1,7^{\mathrm{ab}}$ & $5,8 \pm 0,2^{\mathrm{a}}$ & $1,8 \pm 0,1^{\mathrm{a}}$ & $1,7 \pm 0,1^{\mathrm{ab}}$ \\
\hline $\begin{array}{l}\text { Charolês }(\mathrm{C}) \\
\text { Charolais }(C)\end{array}$ & $33,8 \pm 1,4^{\mathrm{b}}$ & $34,1 \pm 1,3^{\mathrm{c}}$ & $6,7 \pm 0,2^{b}$ & $1,5 \pm 0,1^{b}$ & $1,5 \pm 0,1^{\mathrm{a}}$ \\
\hline $\begin{array}{l}\text { Nelore } \times \mathrm{A} \\
\text { Nellore } \times A\end{array}$ & $41,2 \pm 1,3^{\mathrm{a}}$ & $42,3 \pm 1,2^{\mathrm{a}}$ & $5,9 \pm 0,2^{\mathrm{a}}$ & $1,7 \pm 0,0^{\mathrm{a}}$ & $1,8 \pm 0,0^{\mathrm{b}}$ \\
\hline $\begin{array}{l}\text { Nelore } \times \mathrm{C} \\
\text { Nellore } \times C\end{array}$ & $35,4 \pm 1,3^{\mathrm{b}}$ & $37,1 \pm 1,2^{b c}$ & $6,5 \pm 0,2^{\mathrm{b}}$ & $1,6 \pm 0,1^{\mathrm{ab}}$ & $1,7 \pm 0,0^{\mathrm{ab}}$ \\
\hline \multicolumn{6}{|l|}{$\begin{array}{l}\text { Sexo do bezerro } \\
\text { Calf Sex }\end{array}$} \\
\hline $\begin{array}{l}\text { Macho } \\
\text { Male }\end{array}$ & $39,7 \pm 1,0$ & $40,1 \pm 1,0^{\mathrm{a}}$ & $6,0 \pm 0,1^{\mathrm{a}}$ & $1,7 \pm 0,0^{\mathrm{a}}$ & $1,7 \pm 0,0 \mathrm{a}$ \\
\hline $\begin{array}{l}\text { Fêmea } \\
\text { Female }\end{array}$ & $36,9 \pm 1,0$ & $36,7 \pm 0,9^{\mathrm{b}}$ & $6,4 \pm 0,1^{b}$ & $1,6 \pm 0,0^{\mathrm{b}}$ & $1,6 \pm 0,0^{\mathrm{b}}$ \\
\hline $\begin{array}{l}\text { Médias, na coluna, para } \\
\text { Means, within a column, for } \\
1 \text { EPPARTO = (P205/PVP)" } \\
\text { P205/(PVP) })^{0,75} \text {. }\end{array}$ & $\begin{array}{l}\text { mo item, seguida } \\
\text { ee item, followed by } \\
\text { DESMAME = (P2 }\end{array}$ & $\begin{array}{l}\text { or letras diferentes } \\
\text { erent letters are differen } \\
\text { PVD) }{ }^{*} 100 ; E P N D T=N\end{array}$ & $\begin{array}{l}\text { erentes }(\mathrm{P}<0 \\
\text { 05) by } t \text { test. } \\
\text { DTAL/P205; } \mathrm{E}\end{array}$ & $\begin{array}{l}\text { elo teste t. } \\
\text { ARTO=P205/(P }\end{array}$ & 75;EPMBDES \\
\hline
\end{tabular}

$1 / 2 \mathrm{~A}$. Angus) com vacas que amamentaram bezerros puros (Charolês + A. Angus), observou-se que o primeiro grupo desmamou $9,38 \%$ mais quilos de bezerros por unidade de peso metabólico de vaca ao parto, e produziram mais quilos de bezerros $(7,0 \%)$ para cada $100 \mathrm{~kg}$ de vacas ao desmame. De maneira geral, vacas com bezerros foram mais eficientes do que vacas com bezerras, similarmente ao que foi encontrado por KRESS et al. (1990), EUCLIDES FILHO et al. (1995), ALENCAR et al. (1997) e ALENCAR et al. (1999ab). Isto ocorreu porque os bezerros foram mais pesados que as bezerras e por não ter havido diferença nos pesos e na produção de leite das vacas amamentando machos e fêmeas.

Os melhores resultados para as vacas Aberdeen Angus, vacas com bezerros mestiços e vacas com bezerros machos mostraram que vacas mais leves e ou com bezerros que tiveram bom desenvolvimento, foram as que apresentaram melhor produtividade. Este fato foi observado por EUCLIDES FILHO et al. (1995), os quais demonstraram que as vacas Fleckvieh-Nelore (as mais eficientes) foram mais leves ao desmame, porém apresentaram bezerros mais pesados que as vacas Chianina-Nelore e Charolês-Nelore. Os dados observados neste trabalho foram similares aos encontrados por estes autores, 41 e $38 \mathrm{~kg}$ de bezerros por $100 \mathrm{~kg}$ de vacas. No entanto, KRESS et al. (1990) observaram que vacas de tamanho médio foram superiores. Por outro lado, MONTAÑOBERMUDEZ et al. (1990) e MONTAÑOBERMUDEZ e NIELSEN (1990), comparando eficiência de produção pela relação quilos de bezerros desmamados e requerimento total de energia, observaram que as vacas mais pesadas e com menor produção de leite foram mais eficientes. Todavia, o grupo que produziu menos leite registrou em média $8,5 \mathrm{~kg}$ de leite diariamente, valor bem acima dos observados na literatura nacional para gado de corte.

Os resultados para peso das vacas ao parto e ao desmame, peso dos bezerros ao desmame, produção de leite e requerimentos de energia, segundo os tratamentos (pastagens) a que os animais foram submetidos, são apresentados na Tabela 3. A parição ocorreu de final de agosto a início de novembro e, consequientemente, as vacas que receberam pastagem cultivada por um período maior (T2 e T4) foram mais pesadas ao parto; porém, a suplementação alimentar com acesso à pastagem por duas horas diárias (T3), durante os meses de julho e agosto, não teve efeito significativo sobre o peso ao parto, quando 
comparado com as vacas que estavam apenas no campo natural (T1). Apesar de, após o início de novembro, todas as vacas terem sido submetidas apenas à pastagem natural e o desmame ter ocorrido em maio, os pesos das vacas ao desmame seguiram a mesma tendência dos pesos ao parto, porém não foram diferentes estatisticamente.

As vacas que tiveram maior tempo de acesso à pastagem cultivada (T4) produziram mais leite do que aquelas que permaneceram todo o tempo em pastagem natural (T1), enquanto as dos outros tratamentos tiveram produções intermediárias e não diferiram das demais. As vacas que foram mais pesadas ao parto e produziram mais leite tiveram, por conseguinte, maiores exigências em NDT, já que esta estimativa teve como base esses dois parâmetros. Os bezerros mais pesados foram provenientes de mães que tiveram maior acesso à pastagem cultivada e os mais leves, daquelas alimentadas em campo natural, refletindo de certa maneira a maior e menor produção de leite materno. Estes resultados concordam com POLLI e LOBATO (1985), que observaram maior produção de leite e maior peso dos bezerros ao desmame em vacas mantidas em pastagem temperada (azevém + trevo vesiculoso), por um período de 60 dias pós-parto, comparadas àquelas mantidas em campo natural. Do mesmo modo, LOBATO et al. (1998) observaram que vacas em pastagem natural, suplementadas nos períodos pré e pós-parto, apresentaram bezerros $7 \mathrm{~kg}$ mais pesados a desmama do que bezerros de vacas não-suplementadas. De modo geral, as produções de leite e os pesos dos bezerros podem ser considerados baixos, devido à baixa disponibilidade e qualidade do campo natural. Vale ressaltar que, além da baixa condição da pastagem natural durante o inverno e início de primavera, durante o verão, ocorreu seca, que prejudicou sensivelmente a disponibilidade da pastagem (RIBEIRO et al., 1990). Com raças e pastagens distintas, ALBUQUERQUE et al. (1993) também obtiveram baixas produções leiteiras, decorrentes do período de seca durante a lactação. As correlações simples entre produção média diária de leite das vacas das raças Charolês e Aberdeen Angus e o peso dos seus bezerros aos 205 dias de idade, foram, respectivamente, 0,69 e $0,71 \quad(\mathrm{P}<0,01)$. ROBISON et al. (1978) e ALBUQUERQUE et al. (1993), também, encontraram correlações positivas entre pesos ao desmame e produção de leite das mães.

O desempenho reprodutivo é de suma importância nas comparações de produtividade (EUCLIDES FILHO et al., 1984; MONTAÑO-BERMUDEZ e NIELSEN, 1990; BARCELLOS et al., 1996). Vacas adequadamente alimentadas nos períodos pré e pós-parto apresentam maiores taxa de prenhez e produção de leite. Para taxas adequadas de prenhez, é necessário ganho de peso no período pós-parto (ROVIRA, 1974). Mesmo pequenas perdas de peso, desde que os pesos ao parto sejam bons, não prejudicam o desempenho reprodutivo (LOBATO et al., 1998). Os tratamentos alimentares não tiveram o efeito esperado sobre o desempenho reprodutivo, no qual, em função de perdas de peso $(0,195 \mathrm{~kg}$ por dia) do parto ao final da monta, foram obtidas taxas de prenhez que variaram de 0,0 a $11,1 \%$ (P>0,05; RIBEIRO et al., 1990). Dessa maneira, o desempenho reprodutivo não foi utilizado em medidas de eficiência produtiva. Resultados da eficiência produtiva, segundo os tratamentos, são apresentados na Tabela 4. De modo geral, pode-se observar que as vacas que receberam pasta-

Tabela 3 - Médias estimadas e erros-padrão para pesos das vacas ao parto (PVP) e ao desmame (PVD), peso dos bezerros ao desmame (P205), produção média diária de leite ajustada para $4 \%$ de gordura e requerimento de energia da vaca até o desmame (NDTTOTAL), segundo o tratamento

Table 3 - Least square means and standard errors for cow weight at calving (CWC) and at weaning (CWW), calves weaning weight (W205), daily average milk production adjusted to $4 \%$ of fat and cow energy requirement until weaning (TDNTOTAL), according to treatment

\begin{tabular}{lccccc}
\hline Item & $\begin{array}{c}\text { PVP, } \mathrm{kg} \\
C W C\end{array}$ & $\begin{array}{c}\text { PVD, } \mathrm{kg} \\
C W W\end{array}$ & $\begin{array}{c}\text { P205, kg } \\
\text { Tratamento }\end{array}$ & $\begin{array}{c}\text { LEITE-4\%, litros } \\
\text { MILK-4\%, liters }\end{array}$ & $\begin{array}{c}\text { NDTTOTAL, kg } \\
\text { TDNTOTAL }\end{array}$ \\
\hline Treatment & & & & & \\
T1 & & & & \\
T2 & $345,8 \pm 11,4 \mathrm{a}$ & $358,0 \pm 10,6$ & $121,5 \pm 4,5 \mathrm{a}$ & $2,9 \pm 0,2 \mathrm{a}$ & $781,4 \pm 25,0^{\mathrm{a}}$ \\
T3 & $372,7 \pm 9,4 \mathrm{ab}$ & $360,4 \pm 8,8$ & $133,0 \pm 3,8 \mathrm{ab}$ & $3,4 \pm 0,2 \mathrm{ab}$ & $858,2 \pm 20,7^{\mathrm{bc}}$ \\
T4 & $343,1 \pm 13,6 \mathrm{a}$ & $347,2 \pm 12,6$ & $138,0 \pm 5,4 \mathrm{bc}$ & $3,1 \pm 0,3 \mathrm{ab}$ & $800,6 \pm 29,8^{\mathrm{ab}}$ \\
\hline
\end{tabular}

Médias, na coluna, seguidas por letras diferentes são diferentes $(P<0,05)$ pelo teste $t$.

Means, within a column, followed by different letters are different $(P<.05)$ by $t$ test. 
Rev. bras. zootec.

Tabela 4 - Médias estimadas e erros-padrão para medidas de eficiência produtiva ${ }^{1}$ de vacas de corte, segundo o tratamento Table 4 - Least square means and standard errors for productive efficiency ${ }^{1}$ of beef cows according to treatment

\begin{tabular}{lccccc}
\hline Item & $\begin{array}{c}\text { EPPARTO, kg } \\
\text { CALVINGPE }\end{array}$ & $\begin{array}{c}\text { EPDESMAME, kg } \\
\text { WEANINGPE }\end{array}$ & $\begin{array}{c}\text { EPNDT, kg } \\
\text { TDNPE }\end{array}$ & $\begin{array}{c}\text { EPMBPARTO } \\
C A L V I N G M B P E\end{array}$ & $\begin{array}{c}\text { EPMBDESMAME } \\
W E A N I N G M B P E\end{array}$ \\
\hline $\begin{array}{l}\text { Tratamento } \\
\text { Treatment }\end{array}$ & & & & \\
T1 & $36,9 \pm 1,5$ & $34,8 \pm 1,4^{\mathrm{a}}$ & $6,5 \pm 0,2^{\mathrm{a}}$ & $1,5 \pm 0,1^{\mathrm{a}}$ & $1,5 \pm 0,1^{\mathrm{a}}$ \\
T2 & $36,5 \pm 1,2$ & $37,4 \pm 1,1^{\mathrm{ab}}$ & $6,5 \pm 0,1^{\mathrm{a}}$ & $1,6 \pm 0,0^{\mathrm{ab}}$ & $1,6 \pm 0,0^{\mathrm{ab}}$ \\
T3 & $40,5 \pm 1,8$ & $40,1 \pm 1,6^{\mathrm{bc}}$ & $5,8 \pm 0,2^{\mathrm{b}}$ & $1,7 \pm 0,1^{\mathrm{b}}$ & $1,7 \pm 0,1^{\mathrm{bc}}$ \\
T4 & $39,3 \pm 1,4$ & $41,2 \pm 1,3^{\mathrm{c}}$ & $6,1 \pm 0,2^{\mathrm{ab}}$ & $1,7 \pm 0,1^{\mathrm{b}}$ & $1,8 \pm 0,1^{\mathrm{c}}$ \\
\hline
\end{tabular}

Médias, na coluna, seguidas por letras diferentes são diferentes $(P<0,05)$ pelo teste t.

Means, within a column, followed by different letters are different $(P<.05)$ by $t$ test.

1 EPPARTO $=(\text { P205/PVP })^{*} 100 ;$ EPDESMAME $=(\text { P205/PVD })^{*} 100 ;$ EPNDT $=$ NTDTOTAL/P205; EPMBPARTO $=$ P205/(PVP $)^{0,75} ;$ EPMBDESMAME $=$ $\mathrm{P} 205 /(\mathrm{PVP})^{0,75}$

1 CALVINGPE $=(\text { W205/CWC })^{*} 100 ;$ WEANINGPE $=(\text { W205/CWW })^{*} 100 ;$ TDNPE $=$ TDNTOTALW205; CALVINGMBPE $=$ W205/(CWC $)^{75} ;$ WEANINGMBPE $=$ W205/(CWW $)^{75}$

gem cultivada por um período maior de tempo (T4) foram as mais eficientes; contrariamente, as que permaneceram apenas em pastagem natural (T1) foram menos eficientes. Os tratamentos que propiciaram um período intermediário de acesso à pastagem cultivada apresentaram resultados intermediários. Estes resultados mostram que a pastagem natural não estava atendendo as exigências dos animais para seu potencial ótimo de produção. SIMEONE e LOBATO (1996) e QUADROS e LOBATO (1997) verificaram que, em taxa de lotação menor, há maior desempenho produtivo do rebanho de cria $(0,6$ vs 0,85 ou 0,8 equivalente vaca).

As cinco medidas de eficiência produtiva avaliadas apresentaram valores de correlação simples, que podem ser considerados altos (Tabela 5). Os valores absolutos variaram de 0,82 a 0,99 para a raça Charolês e de 0,57 a 0,98 para a raça Aberdeen Angus. Os valores próximos a unidade referem-se a correlações entre as duas medidas usando pesos ao parto (EPPARTO e EPMBPARTO) ou entre as duas medidas usando pesos ao desmame (EPDESMAME e EPMBDESMAME). As menores correlações ocorreram entre a medida que utilizou o peso metabólico da vaca ao desmame e a aquela que usou o requerimento de energia. Neste último caso, estes menores valores podem ser explicados, parcialmente, pela utilização do peso da vaca ao parto para estimar a exigência em energia, e não o peso ao desmame. Como as correlações indicam, quando forem usados pesos das vacas ao parto ou ao desmame, é possível haver diferentes classificações para os mesmos ani-

Tabela 5 - Correlações simples ${ }^{1}$ entre as diferentes medidas de eficiência produtiva ${ }^{2}$

Table 5 - Simple correlations ${ }^{1}$ among the different productive efficiency measurements ${ }^{2}$

\begin{tabular}{|c|c|c|c|c|c|}
\hline Item & $\begin{array}{l}\text { EPPARTO, } \mathrm{kg} \\
C A L V I N G P E\end{array}$ & $\begin{array}{l}\text { EPDESMAME, kg } \\
\text { WEANINGPE }\end{array}$ & $\begin{array}{c}\text { EPNDT, } \mathrm{kg} \\
T D N P E\end{array}$ & $\begin{array}{l}\text { EPMBPARTO } \\
C A L V I N G M B P E\end{array}$ & $\begin{array}{l}\text { EPMBDESMAME } \\
\text { WEANINGMBPE }\end{array}$ \\
\hline $\begin{array}{l}\text { EPPARTO } \\
C A L V I N G P E\end{array}$ & - & $0,74 * * 0,90 * *$ & $-0,83 * *-0,89 * *$ & $0,97 * * 0,98 * *$ & $0,72 * * 0,88 * *$ \\
\hline $\begin{array}{l}\text { EPDESMAME } \\
\text { WEANINGPE }\end{array}$ & & - & $-0,59 * *-0,82 * *$ & $0,81 * * 0,93 * *$ & $0,98 * * 0,99 * *$ \\
\hline $\begin{array}{l}\text { EPNDT } \\
T D N P E\end{array}$ & & & - & $-0,80 * *-0,89 * *$ & $-0,57 * *-0,82 * *$ \\
\hline $\begin{array}{l}\text { EPMBPARTO } \\
C A L V I N G M B P E \\
\text { EPMBDESMAME } \\
W E A N I N G M B P E\end{array}$ & & & & - & $\begin{array}{c}0,83 * * 0,94 * * \\
-\end{array}$ \\
\hline $\begin{array}{ll}* * & (\mathrm{P}<0,01) . \\
1 & \text { Os primeiros valore } \\
2 & \text { EPPARTO = (P20 } \\
& \text { EPMBDESMAME = } \\
* * & (P<.01) . \\
1 & \text { First values are for Ab } \\
& \text { Fre }\end{array}$ & $\begin{array}{l}\text { vacas Aberdeen } \\
0 ; \text { EPDESMAME } \\
0,75 \text {. } \\
\text { s cows and second } v\end{array}$ & $\begin{array}{l}\text { ngus e os segundos } p \\
=(P 205 / P V D)^{*} 100 ; \mathrm{E} \\
\\
\text { ues are for Charolais cows }\end{array}$ & $\begin{array}{l}\text { oara vacas Charolês. } \\
\text { EPNDT = NTDTOTA }\end{array}$ & LL/P205; EPMBPART & $T O=P 205 /(P V P)^{0,75}$ \\
\hline
\end{tabular}


mais. Estes resultados foram similares aos observados por KRESS et al. (1990).

Os resultados encontrados neste trabalho são válidos para a condição alimentar observada, a qual não foi considerada boa. Em melhores condições de alimentação e com grupos genéticos distintos, os resultados podem ser diferentes.

\section{Conclusões}

Vacas primíparas da raça Charolês produziram mais leite e bezerros mais pesados ao desmame, contudo foram menos produtivas, nas diferentes medidas de eficiência avaliadas, que as vacas Aberdeen Angus.

Vacas com bezerros machos e mestiços foram mais produtivas.

A utilização de pastagens cultivadas de inverno, nos períodos de carência da pastagem natural, pode resultar em maior produção de leite, bezerros mais pesados ao desmame e maior produtividade do gado de cria.

\section{Referências Bibliográficas}

ALBUQUERQUE, L.G., ELER, J.P., COSTA, M.J.R.P. et al. 1993. Produção de leite e desempenho do bezerro na fase de aleitamento em três raças bovinas de corte. R. Bras. Zootec., 22(5):745-754.

ALENCAR, M.M., TREMATORE, R.L., OLIVEIRA, J.A.L. et al. 1997. Desempenho produtivo de vacas da raça Nelore e cruzadas Charolês x Nelore, Limousin x Nelore e Tabapuã x Gir. R. Bras. Zootec., 26(3):467-472.

ALENCAR, M.M., OLIVEIRA, J.A.L., ALMEIDA, M.A. 1999a. Idade ao primeiro parto, peso ao parto e desempenho produtivo de vacas Nelores e cruzadas Charôles 'Nelore. R. Bras. Zootec., 28(4):681-686.

ALENCAR, M.M., TULLIO, R.R., CORRÊA, L.A. 1999b. Pesos e relações de peso de bezerros filhos de vacas Nelore e cruzadas Canchim $\times$ Nelore. R. Bras. Zootec., 28(5):968-973.

BARCELLOS, J.O.J., LOBATO, J.F.P., FRIES, L.A. 1996. Eficiência de vacas primíparas Hereford e cruzas Hereford-Nelore acasaladas no outono/inverno ou na primavera/verão. R. Bras. Zootec., 25(3):414-427.

BUSTAMANTE, J.R.B., FONSECA, F.A., FONTES, C.A.A. et al. 1997. Efeito da condição corporal ao parto e da amamentação na eficiência reprodutiva de vacas da raça Nelore. R. Bras. Zootec., 26(6):1090-1095.

CAMPOS, J. 1995. Tabelas para cálculo de rações. 2.ed. Viçosa: UFV. 64p.

CRUZ, G.M., ALENCAR, M.M., TULLIO, R.R. 1997. Produção e composição do leite de vacas das raças Canchim e Nelore. R. Bras. Zootec., 26(5):887-893.

EUCLIDES FILHO, K., RESTLE, J., OLSON, T.A. et al. Medidas de eficiência na produção de terneiros a partir de vacas de tamanho e habilidade leiteira diferentes. In: REUNIÃO ANUAL DA SOCIEDADE BRASILEIRA DEZOOTECNIA, 21, 1984, Belo Horizonte. Anais... Belo Horizonte: SBZ, 1984. p.138.

EUCLIDES FILHO, K., FIGUEIREDO, G.R., EUCLIDES, V.P.B 1995. Eficiência de produção de vacas de corte com diferentes potenciais para produção de leite. Pesq. Agropec. Bras., 30(7):1003-1007.

KRESS, D.D., DOORNBOS, D.E., ANDERSON, D.C. 1990. Performance of crosses among Hereford, Angus and Simmental cattle with different levels of Simmental breeding: V. Calf production, milk production and reproduction of three- to eightyear-old dams. J. Anim. Sci., 68(7):1910-1921.

LEAL, T.C., FREITAS, J.E. 1982. Correlação entre produção de leite e ganho de peso de terneiros da raça charolesa. Anuário Técnico do IPZFO, 9:91-101.

LOBATO, J.F.P., DERESZ, F., LEBOUTE, E.M. et al. 1998 Pastagens melhoradas e suplementação alimentar no comportamento reprodutivo de vacas de corte primíparas. R. Bras. Zootec., 27(1):47-53.

MELTON, A.A., RIGGS, J.K., NELSON, L.A. et al. 1967. Milk production, composition and calf gains of Angus, Charolais and Hereford cows. J. Anim. Sci., 26(4):804-809.

MONTAÑO-BERMUDEZ, M., NIELSEN, M.K., DEUTSCHER, G.H. 1990. Energy requirements for maintenance of crossbred beef cattle with different genetic potential for milk. J. Anim. Sci., 68(8):2279-2288.

MONTAÑO-BERMUDEZ, M., NIELSEN, M.K. 1990. Biological efficiency to weaning and to slaughter of crossbred beef cattle with different genetic potential for milk. J.Anim. Sci., 68(8):2297-2309.

POLLI, V.A., LOBATO, J.F.P. Utilização de pastagem temperada por diferentes categorias do rebanho. In: REUNIÃO ANUAL DA SOCIEDADE BRASILEIRA DE ZOOTECNIA, 22, 1985, Balneário Camboriú. Anais... Viçosa: SBZ, 1985. p.411.

PRICHARD, D.L., MARSHALL, T.T. Effects of cow size and milk production on nutrientrequirements. In: ANNUALFLORIDABEEF CATTLESHORTCOURSE, 42, 1993, Gainesville. Proceedings... Gainesville, USA: University of Florida, 1993. p.28-33.

QUADROS, S.A.F., LOBATO, J.F.P. 1997. Efeitos da lotação animal na produção de leite de vacas de corte primíparas e no desenvolvimento de seus bezerros. R. Bras. Zootec., 26(1):27-33.

REYNOLDS, W.L., DE ROUEN, T.M., BELLOWS, R.A. 1978. Relationships of milk yield of dam to early growth rate of straightbred and crossbred calves. J. Anim. Sci., 47(3):584-594.

RIBEIRO, E.L.A., RESTLE, J., PIRES, C.C. 1990. Influência de diferentes sequiências de pastagens no peso e no desempenho reprodutivo de vacas de corte, de dois grupos genéticos, com primeira cria ao pé. Semina, 11(1):24-32.

RIBEIRO, E.L.A., RESTLE, J. 1991. Desempenho de terneiros Charolês e Aberdeen Angus puros e seus mestiços com Nelore. Pesq. Agropec. Bras., 26(8):1145-1151.

RIBEIRO, E.L.A., RESTLE, J., PIRES, C.C. 1991. Produção e composição do leite em vacas Charolês e Aberdeen Angus amamentando terneiros puros ou mestiços. Pesq. Agropec. Bras., 26(8):1267-1273.

ROBISON, O.W., YUSUFF, M.K.M., DILLARD, E.U. 1978. Milk production in Hereford cows. I. Means and correlations. J. Anim. Sci., 47(1):131-136.

SAS/STAT User's guide. 1989. Version 6, 4.ed. Cary: SAS Institute, v. 2, 846 p.

SIMEONE, A., LOBATO, J.F.P. 1996. Efeitos da lotação animal em campo nativo e do controle da amamentação no comportamento reprodutivo de vacas de corte primíparas. R. Soc. Bras. Zootec., 25(6):1216-1227.

Recebido em: 29/12/99 Aceito em: 22/08/00 